\title{
Effectiveness of adjustable dorsiflexion night splint in combination with accommodative foot orthosis on plantar fasciitis
}

\author{
Winson C. C. Lee, PhD; ${ }^{1}$ W. Y. Wong, PhD; ${ }^{1-2}$ Eddy Kung, MSc; ${ }^{2}$ Aaron K. L. Leung, PhD ${ }^{1^{*}}$ \\ ${ }^{1}$ Department of Health Technology and Informatics, The Hong Kong Polytechnic University, Kowloon, Hong Kong; \\ ${ }^{2}$ Prosthetic and Orthotic Department, Tuen Mun Hospital, New Territories, Hong Kong
}

\begin{abstract}
Foot orthoses and night splints have been used separately to treat patients with plantar fasciitis, but were not always successful. Combined use of both orthoses might give better outcomes. This study evaluated the effectiveness of a soft and self-adjustable dorsiflexion night splint in combination with an accommodative foot orthosis for patients with plantar fasciitis. Twenty-eight patients were assigned to group A (foot orthosis only) and group B (combination of foot orthosis and dorsiflexion night splints). A Foot Function Index (FFI) questionnaire was used to evaluate the pain and functions of feet just before, 2 weeks after, and 8 weeks after the treatments. Results showed that subjects in group B had significantly reduced pain scores at week $2(p<0.001)$ and week $8(p<$ $0.001)$. In group $A$, no statistical differences were noted in the pain $(p=0.15)$, disability $(p=0.56)$, activity limitation $(p=$ $0.75)$, and total FFI $(p=0.35)$ scores for the three time periods. The application of foot orthoses with adjustable dorsiflexion night splints was found to be more effective than the application of foot orthoses alone in relieving foot pain in patients with plantar fasciitis.
\end{abstract}

Clinical Trial Registration: Registered at HKU Clinical Trial Register (HKCTR-1318); http://www.HKClinicalTrials.com

Key words: dorsiflexion, foot, Foot Function Index, heel pain, night splints, orthoses, orthotics, pain, plantar fasciitis, rehabilitation.

\section{INTRODUCTION}

About 10 percent of the general population have experienced heel pain at some stage in their lives [1]. One of the most common causes is plantar fasciitis, which may result from repetitive and excessive loads on the fascia [2-4]. The management of plantar fasciitis can be classified into conservative and surgical approaches. Surgical intervention, including plantar fasciotomy and plantar fascia release, can be indicated when conservative treatment fails to treat the heel pain after 12 mo [5].

Foot orthotic intervention is one commonly used conservative approach for plantar fasciitis, and its effectiveness has been studied based on the subjective feedback of patients [6]. Lynch et al. suggested that orthotic insoles were more effective in relieving pain than other conservative treatments, such as antiinflammatory medication, stretching, and strengthening exercises [7]. Although offthe-shelf orthotic insoles and customized foot orthoses were found equally effective in relieving pain in plantar fasciitis in some studies [8], some other investigations did

\footnotetext{
Abbreviations: $\mathrm{ANOVA}=$ analysis of variance, $\mathrm{BMI}=$ body mass index, FFI $=$ Foot Function Index, PPT $=$ Professional Protective Technology.

*Address all correspondence to Aaron K. L. Leung, PhD; Interdisciplinary Division of Biomedical Engineering, The Hong Kong Polytechnic University, Hung Hom, Kowloon, Hong Kong; 852-2766-7676; fax: 852-2334-2429.

Email: htaaron@polyu.edu.hk

http://dx.doi.org/10.1682/JRRD.2011.09.0181
} 
not agree that orthoses could reduce the self-reported pain associated with plantar fasciitis [9-10]. The inconsistency could be due to the use of different orthotic designs [11]. Accommodative and functional orthoses are commonly used. Accommodative orthoses are made of soft and elastic materials aiming to provide cushioning and reduce the peak plantar pressure [12]. Functional orthoses realign the foot in an optimal position during weight bearing. Both types of orthoses can be off the shelf and custom made, and functional orthoses are usually more expensive.

When treating plantar fasciitis, some clinicians opt to use a dorsiflexion night splint, which maintains the ankle at a neutral or dorsiflexed position during sleep. This restores the flexibility of the calf and plantar muscles as well as reduces the tension stress on plantar fascia during the first few steps in the morning [13-18], which could reduce the pain associated with plantar fasciitis throughout the day. The splint is usually made of thermoplastics. Some previous studies suggested that dorsiflexion night splints effectively relieve plantar fasciitis $[1,16]$ and that their treatment effects were comparable to customized and off-the-shelf foot orthoses [4,19]. However, some other studies reported that the night splints could easily cause discomfort at the feet and thus lower the quality of sleep [20] and compliance rates and affect treatment outcomes [21]. The discomfort is caused by the high pressure applied from the thermoplastics to the malleolus and the back of the heel when the feet rest on the bed during sleep. Some design changes in improving the comfort of the use of a night splint are required.

Orthotic insoles and dorsiflexion night splints work in different ways, with insoles reducing the peak plantar pressure and dorsiflexion night splints restoring the flexibility of soft tissues. This led to the hypothesis that combining the use of foot orthoses and night splints might improve pain relief for plantar fasciitis. While the combination approach was used in some clinical centers, the treatment outcomes were not well documented. In this study, we evaluated whether the combination of foot orthoses and dorsiflexion night splints made of elastic material and adjustable straps could provide better treatment outcomes than using accommodative foot orthoses alone.

\section{METHODS}

This study was conducted at Tuen Mun Hospital (Hong Kong). Inclusion criteria were patients older than
18 who were diagnosed with plantar fasciitis by licensed physicians and presenting symptoms of plantar heel pain during the first few steps in the morning and prolonged walking as well as tenderness over the medial tubercle of the calcaneus. They did not receive any prior foot orthotic intervention, steroid injection, or surgical treatments and did not have a history of trauma or fracture. All subjects had to be able to complete a Foot Function Index (FFI) questionnaire independently.

The subjects (Table 1) were patients referred to the Prosthetic and Orthotic Department of Tuen Mun Hospital for orthotic treatment of plantar fasciitis. A consecutive sampling approach was used in which all incoming patients who met the inclusion criteria and quota requirements were included as subjects. Subjects were assigned to one of two groups: subjects in group A received an accommodative foot orthosis and subjects in group B received the same type of foot orthosis in combination with an adjustable dorsiflexion night splint. Subjects were assigned to treatment groups when they were enrolled into the study, with the first two eligible patients assigned to group $\mathrm{A}$ and the third and fourth assigned to group B. Body mass index (BMI) of each subject was measured. Normal weight was defined as BMI between 17 and $25 \mathrm{~kg} / \mathrm{m}^{2}$, and overweight was defined as BMI greater than $25 \mathrm{~kg} / \mathrm{m}^{2}$. Each treatment group had quotas of seven normal weight and seven overweight subjects, because body weight might affect treatment outcomes. This sample size would produce a statistical power of 0.8 , assuming a medium effect size of 0.6 [22] and onesided significance level of 0.05 on a repeated-measures design. Consecutive sampling was continued and the same subject assignment method was used for subsequent patients until each subject quota was full. If a particular weight category of a treatment group was full, the subject would be assigned to the other treatment group. A patient would not be included in this study if the quota of the corresponding weight category in both treatment groups was full.

Each subject completed a standard FFI questionnaire just before the interventions (baseline) and during the clinical follow-ups 2 weeks and 8 weeks after being prescribed the orthoses. The subjects placed a mark on a $10 \mathrm{~cm}$ line with word anchors on both sides representing two extremes of answers to each question. The questionnaire was shown to have high test-retest reliability and internal consistency in an earlier study [23]. If both feet had symptoms of pain, only the more painful foot would 
Table 1.

Demographic profile of participants with plantar fasciitis in group A (foot orthosis only) and group B (foot orthosis and dorsiflexion night splint).

\begin{tabular}{|c|c|c|c|c|c|c|c|c|}
\hline Group & Subject & Sex & $\begin{array}{l}\text { Age } \\
\text { (yr) }\end{array}$ & $\begin{array}{c}\text { BMI } \\
\left(\mathrm{kg} / \mathrm{m}^{2}\right)\end{array}$ & $\begin{array}{l}\text { Duration of Heel Pain } \\
\text { (mo) }\end{array}$ & Baseline Pain & Baseline Total & Pain Side \\
\hline \multirow[t]{9}{*}{$\overline{\mathbf{A}}$} & 1 & $\mathrm{~F}$ & 41 & 26.7 & 6 & 74 & 69 & Left \\
\hline & 2 & $\mathrm{~F}$ & 53 & 19.9 & 6 & 56 & 42 & Right \\
\hline & 4 & $\mathrm{~F}$ & 40 & 23.4 & 6 & 45 & 33 & Left \\
\hline & 5 & $\mathrm{~F}$ & 40 & 25.5 & 4 & 56 & 44 & Left \\
\hline & 8 & $\mathrm{~F}$ & 42 & 21.4 & 10 & 62 & 62 & Left \\
\hline & 9 & $\mathrm{~F}$ & 49 & 25.9 & 9 & 79 & 72 & Left \\
\hline & 10 & $\mathrm{~F}$ & 42 & 22.9 & 2 & 52 & 45 & Left \\
\hline & 11 & $\mathrm{~F}$ & 43 & 26.7 & 4 & 58 & 67 & Left \\
\hline & 12 & $\mathrm{~F}$ & 34 & 23.9 & 6 & 65 & 60 & Left \\
\hline \multirow[t]{10}{*}{$\overline{\mathbf{B}}$} & 15 & $\mathrm{~F}$ & 31 & 26.8 & 2 & 72 & 71 & Right \\
\hline & 16 & $\mathrm{~F}$ & 46 & 22.4 & 9 & 50 & 43 & Right \\
\hline & 17 & $\mathrm{~F}$ & 38 & 25.4 & 9 & 52 & 51 & Right \\
\hline & 18 & $\mathrm{~F}$ & 53 & 23.0 & 12 & 65 & 68 & Left \\
\hline & 19 & $\mathrm{~F}$ & 43 & 22.5 & 2 & 70 & 42 & Left \\
\hline & 20 & M & 41 & 20.4 & 12 & 36 & 37 & Right \\
\hline & 21 & $\mathrm{~F}$ & 54 & 26.3 & 8 & 73 & 64 & Left \\
\hline & 22 & $\mathrm{~F}$ & 51 & 21.6 & 4 & 79 & 72 & Right \\
\hline & 23 & $\mathrm{~F}$ & 42 & 28.6 & 2 & 81 & 62 & Left \\
\hline & 24 & $\mathrm{~F}$ & 30 & 22.0 & 6 & 49 & 44 & Right \\
\hline
\end{tabular}

be studied. The questionnaire contained 23 questions rated by a visual analog scale. Nine questions covered aspects of foot pain, another nine questions assessed the level of disability, and five questions addressed activity limitation. Two additional questions were added to the questionnaire at week 2 and week 8 requesting the subjects to answer "yes" or "no" as to whether they had experienced any discomfort while using the orthoses and whether they had stopped using the orthoses consecutively for more than $2 \mathrm{~d}$ for any reasons.

The full-length foot orthoses were made of Poron material (Rogers Corporations; Rogers, Connecticut) with an additional layer of 6 mm-thick Professional Pro- tective Technology (PPT) material (The Langer Group; Stone, Staffordshire, United Kingdom) added to the heel region as a heel lift to enhance peak pressure reduction and shock absorption. The PPT material was found in a previous study to be the most effective in reducing pressure under the painful areas of the foot among seven commonly used insole materials [12]. The adjustable dorsiflexion night splint contained (1) a 3 mm-thick polypropylene footplate; (2) a tailor-made low-profile soft anklefoot sleeve made of perforated neoprene sheets with appropriate sewing that allowed the footplate to be attached, and (3) an adjustable Velcro strap with a buckle (Figure 1(a)). It was designed for nocturnal use only. The 

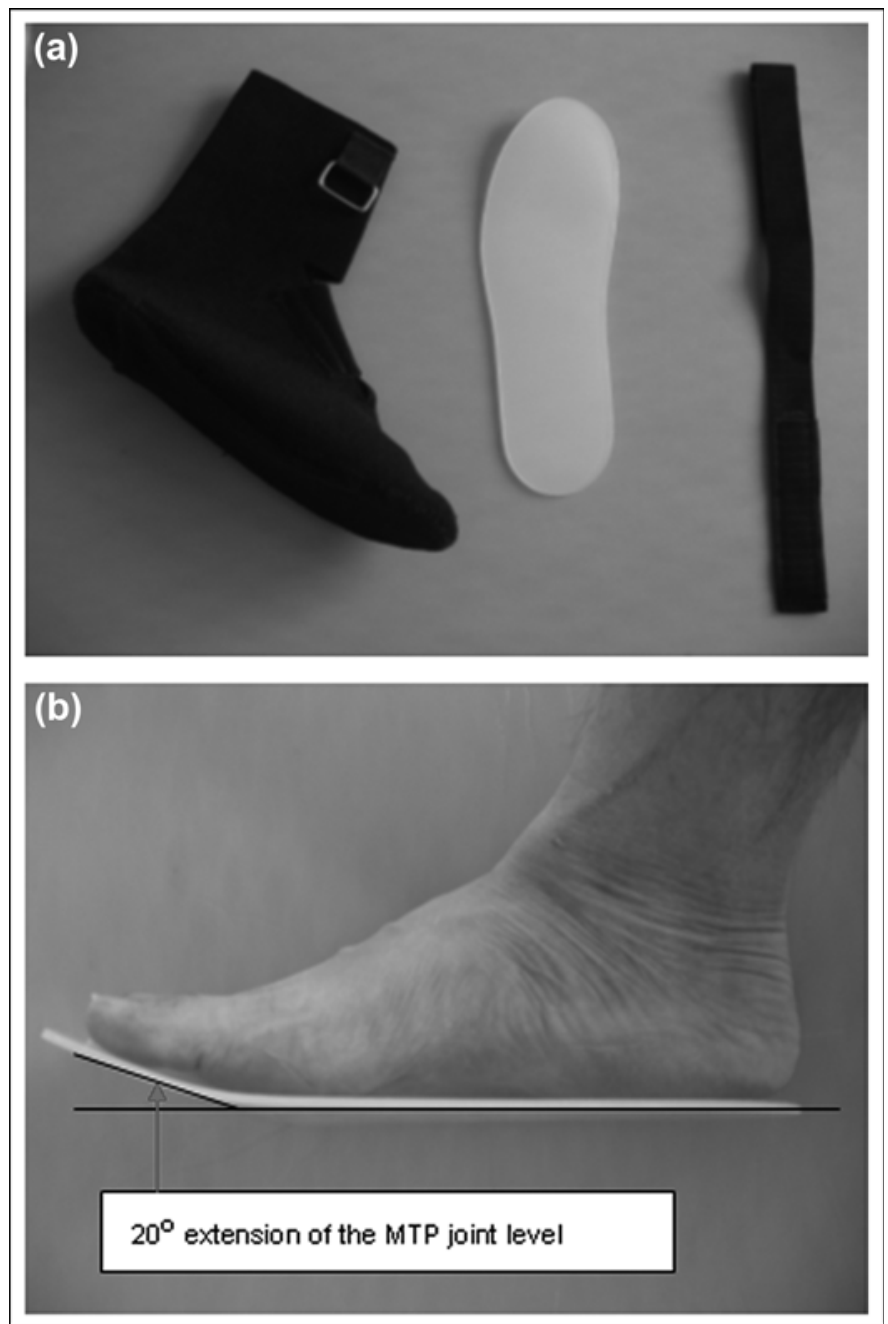

\section{Figure 1.}

(a) Components of dorsiflexion night splint: soft ankle-foot sleeve, footplate, and adjustable Velcro strap with buckle. (b) Polypropylene footplate in night splint extends metatarsophalangeal (MTP) joints.

footplate was bent at $20^{\circ}$ to extend the toes at the metatarsophalangeal joints (Figure 1(b)). The tightness of the Velcro strap, which determined the ankle joint angle, was adjusted so that the ankle was dorsiflexed at $5^{\circ}$ (Figure 2). A marking was made at the strap to ensure the repeatability of strap tension. The subjects were instructed to tighten the strap based on the markings but were allowed to reduce the tension of the straps if required for comfort.

Pain, disability, and activity limitation scores were computed based on the markings on the visual analog scales. Lower scores reflected lower pain, levels of disability, and activity limitation. We calculated total FFI

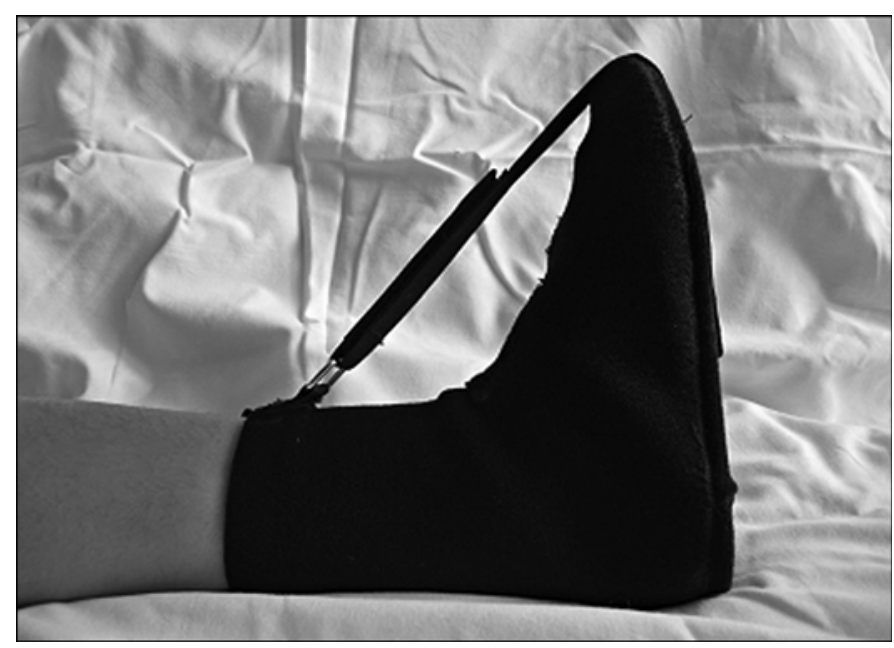

Figure 2.

Dorsiflexion night splint in use.

scores by adding the pain, disability, and activity limitation scores. The person who measured the scores from the visual analog scales did not know to which group the subjects were assigned. Statistical analysis was performed using GraphPad Prism, Version 4 (GraphPad Software, Inc; San Diego, California). One-way repeated-measures analyses of variance (ANOVAs) were performed, separately for groups $\mathrm{A}$ and $\mathrm{B}$, to study whether significant changes in pain, disability, activity limitation, and total FFI scores occurred across the three time sessions. If a significant difference was found in the ANOVA, a pairwise comparison of each of two time sessions using paired $t$-test with Bonferroni corrections was performed. To assess differences between group A and group B, we performed Bonferroni $t$-tests comparing the scores between the two groups at baseline, week 2, and week 8 . The level of significance was set at $\alpha=0.05$, and the confidence interval was 95 percent. When performing multiple comparisons among the three conditions, we performed Bonferroni correction adjusting $\alpha$ to 0.017 .

\section{RESULTS}

None of the subjects had discomfort while using the foot orthoses and night splints or stopped using the orthoses consecutively for more than $2 \mathrm{~d}$ during the 8 weeks. Other than the orthotic treatments given to the subjects, these 28 subjects did not receive any other plantar fasciitis treatments. No statistical differences were found in 
baseline scores for pain $(p=0.64)$, disability $(p=0.44)$, activity limitation $(p=0.35)$, and total FFI $(p=0.96)$ between group A and group B. No statistical differences were found between group A and group B in scores for pain ( $p=0.10)$, disability $(p=0.05)$, activity limitation ( $p=$ $0.13)$, and total FFI $(p=0.06)$ at week 2 . However, the pain score $(p=0.01)$ and total FFI score $(p=0.01)$ in group B were significantly lower than in group A at week 8 .

Table 2 shows the pain, disability, activity limitation, and total FFI scores of subjects in groups A and B for the three time periods. Repeated-measures ANOVA revealed statistical changes in pain scores $(p=0.002)$ and total FFI scores $(p=0.01)$ across the three time sessions in group B. Post-hoc Bonferroni tests showed that group B pain scores at week 8 were significantly lower than at baseline $(-47.0 \%, p<0.001)$ and week $2(-27.7 \%, p=0.006)$. Group B pain scores at week 2 were also significantly lower than at baseline $(-28.8 \%, p<0.001)$. Similar reduction patterns were seen in group B total FFI scores, with scores at week 8 significantly lower than at baseline $(-38.6 \%, p=0.001)$ and week $2(-22.2 \%, p=0.01)$ and total FFI scores at week 2 significantly lower than at baseline $(-21.9 \%, p=0.005)$. The differences in disability $(p=0.06)$ and activity limitation $(p=0.25)$ scores over the three periods of time in group B did not reach statistical significance, although there were notable decreases in the average scores. There were trends of reductions in pain scores $(17.2 \%$ reductions at week 8 compared with baseline) and total FFI scores (12.2\% reductions at week 8 compared with baseline) in group $\mathrm{A}$.

Table 2.

Pain, disability, activity limitation, and total Foot Function Index (FFI) scores (mean \pm standard deviation) for participants with plantar fasciitis in group A (foot orthosis only) and group B (foot orthosis and dorsiflexion night splint) over 8-week study.

\begin{tabular}{lllll}
\hline \multirow{2}{*}{ Group } & \multirow{2}{*}{ Test } & \multicolumn{3}{c}{ Time } \\
\cline { 3 - 5 } & Parameter & Baseline & Week 2 & Week 8 \\
\hline A & Pain & $64 \pm 14$ & $57 \pm 15$ & $53 \pm 14$ \\
& Disability & $57 \pm 21$ & $56 \pm 15$ & $50 \pm 16$ \\
& Activity & $51 \pm 16$ & $52 \pm 15$ & $48 \pm 13$ \\
& Total FFI & $57 \pm 15$ & $55 \pm 12$ & $50 \pm 10$ \\
& Pain & $66 \pm 15$ & $47 \pm 23$ & $34 \pm 26$ \\
& Disability & $56 \pm 19$ & $43 \pm 23$ & $35 \pm 25$ \\
& Activity & $49 \pm 17$ & $44 \pm 20$ & $37 \pm 18$ \\
& Total FFI & $57 \pm 14$ & $45 \pm 20$ & $35 \pm 21$ \\
\hline *Statistical differences $(p<0.05)$ & in repeated-measures analysis of variance.
\end{tabular}

*Statistical differences $(p<0.05)$ in repeated-measures analysis of variance. FFI $=$ Foot Function Index.
However, no statistical differences were noted in the scores for pain $(p=0.15)$, disability $(p=0.56)$, activity limitation $(p=0.75)$, and total FFI $(p=0.35)$ for the three time periods in group $\mathrm{A}$.

\section{DISCUSSION}

During normal walking, at least $10^{\circ}$ of ankle dorsiflexion is required [24]. If the calf muscles are tight, excessive pronation of the foot may occur to compensate for the reduced ankle dorsiflexion. This increases the tensile loads on the plantar fascia. The plantar fascia will experience even higher loads if it is tight as well. Dorsiflexing the ankle and the metatarsophalangeal joints during sleep could reduce the tension of the posterior calf muscles and plantar fascia during daytime walking and, therefore, reduce pain. Poor compliance has been one critical factor affecting the effectiveness of night splints [14-16]. Poor compliance was due to the discomfort and pain caused by overstretching of the calf muscles and the high pressure applied from the thermoplastic component of the splint to the ankle and the back of the heel. The adjustable and soft night splint used in this study addressed these two problems. Subjects could adjust the angle if it created discomfort. In addition, the neoprene material reduced the pressure applied around the ankle and the back of the heel.

During sleep, the ankle is plantar flexed. Although subjects can loosen the tightness of the straps for better comfort, the night splint can still maintain the ankle in a less plantar flexed position. While some previous studies maintained the ankle at neutral positions $[13,20], 5^{\circ}$ of dorsiflexion [4], and nearly maximum dorsiflexion [14], there has been no reported agreement on the optimal ankle position to be maintained with a night splint. This study shows that self-adjustability can avoid discomfort while maintaining functionality. A 3 mm-thick polypropylene footplate was used in this study. A mechanically stronger material might be necessary for obese or more active people. Future studies can also identify whether any relationship exists among the tension of the selfadjusted straps, the angle of the ankle placed within the splint, and the treatment outcome.

The main function of the orthotic insoles used in this study was to reduce the peak pressure under the heel [12], because the elastic insole material deforms at each walking step, increasing the contact area with the foot [25]. These 
orthoses did not require casting of the foot, which reduced the manpower and time of an orthotist and, therefore, reduced production cost. Some functional foot orthoses require foot casting. Such orthoses could realign the foot during weight bearing by providing appropriate arch supports. A previous study suggested that both types of insoles effectively reduced pain scores in 4 weeks [11]. In our study, the accommodative orthotic insole lowered mean pain scores over the 8 weeks; however, a statistical difference was not found. Significant reductions in pain and total FFI scores were achieved when combining the orthotic insole with the self-adjustable night splint.

While findings from previous studies were inconsistent regarding the effectiveness of night splints and orthotic insoles used separately for reducing plantar fasciitis pain $[4,9-11,16,19]$, this study suggests that a treatment protocol combining orthotic insoles with the new night splints was more effective in relieving pain than the orthotic insoles alone. Significant reductions in pain and total FFI scores were found in group B at week 2 and week 8 , but were not seen in group $A$. At week 8 , the pain and total FFI scores in group B were significantly lower than in group A. Because the differences in the baseline pain and total FFI scores between the two groups were small and insignificant, the different treatment outcomes can largely be attributed to the two different treatment methods. The differences in disability and activity limitation scores for the three time periods were insignificant. This could be explained by the lower baseline scores as compared with the baseline pain scores.

Being overweight has been suggested to be a contributing factor to plantar fasciitis, because repetitive and excessive loading can lead to inflammation and consequent pain in the plantar fascia [2-4]. High body weight could deform the PPT material to a larger extent during walking and potentially affect the treatment outcomes. A previous study indicated that an addition of $9.1 \mathrm{~kg}$ of body weight in female subjects increased plantar pressure by more than 10 percent at heel regions [26]. Another study reported that repeated applications of high loading could stiffen insole materials [27], which could affect their ability to reduce peak pressure. The potential effect of body weight on the function of the foot orthosis was the reason why equal numbers of overweight and normal weight subjects were assigned to the two groups. This study did not further investigate whether the treatment outcome would be worse in an overweight subject group, because such an investigation would largely reduce the statistical power. Type of shoe might also affect treatment outcomes. Because the study spanned 8 weeks, however, ensuring that each subject used the same type of shoes over the entire study period was not feasible. This study used a consecutive sampling approach [28] to include patients who met the inclusion criteria within a certain period of time. Random sampling, which allows nonprobability biases to be avoided, was not used because this study followed up new incoming patients referred for orthotic treatments.

When more conservative managements such as orthotic treatments, stretching exercises, and shockwave therapies are unsuccessful in clinical practice, steroid injection is a preferred option [29-30]. Although steroid therapy can significantly reduce pain for short periods in patients with plantar fasciitis [30], a number of complications were reported, such as rupture of the plantar fascia, atrophy of the heel fat pad, and plantar nerve injury secondary to injection [31-34]. This study provides some clues for improving the treatment outcomes of orthoses, which may prevent subsequently more invasive treatments.

\section{CONCLUSIONS}

The application of accommodative foot orthoses with adjustable dorsiflexion night splints was found to be more effective than the application of foot orthoses alone in relieving foot pain in subjects with plantar fasciitis. The soft neoprene material and the self-adjustable strap tension of the soft night splint could avoid discomfort while maintaining the treatment effects.

\section{ACKNOWLEDGMENTS}

\section{Author Contributions:}

Study concept and design: W. C. C. Lee, W. Y. Wong, E. Kung, A. K. L. Leung.

Acquisition of data: E. Kung.

Analysis and interpretation of data: W. C. C. Lee.

Statistical analysis: W. Y. Wong, E. Kung.

Drafting of manuscript: W. Y. Wong.

Critical revision of manuscript for important intellectual content:

W. C. C. Lee, A. K. L. Leung.

Study supervision: A. K. L. Leung.

Financial Disclosures: The authors have declared that no competing interests exist.

Funding/Support: This material was based on work supported by the Department of Health Technology and Informatics, The Hong Kong Polytechnic University. 
Additional Contributions: Winson C. C. Lee and Aaron K. L. Leung are now with the Interdisciplinary Division of Biomedical Engineering, The Hong Kong Polytechnic University, Kowloon, Hong Kong. Institutional Review: Ethical approval was obtained from the Tuen Mun Hospital Ethical Committee and the Human Subject Ethic Subcommittee of The Hong Kong Polytechnic University.

Participant Follow-Up: The authors do not plan to inform participants of the publication of this study.

\section{REFERENCES}

1. Crawford F, Thomson C. Interventions for treating plantar heel pain. Cochrane Database Syst Rev. 2003;(3): CD000416. [PMID:12917892]

2. Aldridge T. Diagnosing heel pain in adults. Am Fam Physician. 2004;70(2):332-38. [PMID:15291091]

3. Cornwall MW, McPoil TG. Plantar fasciitis: etiology and treatment. J Orthop Sports Phys Ther. 1999;29(12):756-60. [PMID:10612073]

4. Martin JE, Hosch JC, Goforth WP, Murff RT, Lynch DM, Odom RD. Mechanical treatment of plantar fasciitis. A prospective study. J Am Podiatr Med Assoc. 2001;91(2): 55-62. [PMID:11266478]

5. Davies MS, Weiss GA, Saxby TS. Plantar fasciitis: how successful is surgical intervention? Foot Ankle Int. 1999; 20(12):803-7. [PMID:10609710]

6. Lee SY, McKeon P, Hertel J. Does the use of orthoses improve self-reported pain and function measures in patients with plantar fasciitis? A meta-analysis. Phys Ther Sport. 2009;10(1):12-18. [PMID:19218074] http://dx.doi.org/10.1016/j.ptsp.2008.09.002

7. Lynch DM, Goforth WP, Martin JE, Odom RD, Preece CK, Kotter MW. Conservative treatment of plantar fasciitis. A prospective study. J Am Podiatr Med Assoc. 1998;88(8): 375-80. [PMID:9735623]

8. Baldassin V, Gomes CR, Beraldo PS. Effectiveness of prefabricated and customized foot orthoses made from lowcost foam for noncomplicated plantar fasciitis: a randomized controlled trial. Arch Phys Med Rehabil. 2009;90(4): 701-6. [PMID:19345789] http://dx.doi.org/10.1016/j.apmr.2008.11.002

9. Landorf KB, Keenan AM, Herbert RD. Effectiveness of foot orthoses to treat plantar fasciitis: a randomized trial. Arch Intern Med. 2006;166(12):1305-10.

[PMID:16801514] http://dx.doi.org/10.1001/archinte.166.12.1305

10. Roos E, Engström M, Söderberg B. Foot orthoses for the treatment of plantar fasciitis. Foot Ankle Int. 2006;27(8): 606-11. [PMID:16919213]

11. Rome K, Gray J, Stewart F, Hannant SC, Callaghan D, Hubble J. Evaluating the clinical effectiveness and costeffectiveness of foot orthoses in the treatment of plantar heel pain: a feasibility study. J Am Podiatr Med Assoc. 2004;94(3):229-38. [PMID:15153583]

12. Leber C, Evanski PM. A comparison of shoe insole materials in plantar pressure relief. Prosthet Orthot Int. 1986; 10(3):135-38. [PMID:3808918]

13. Barry LD, Barry AN, Chen Y. A retrospective study of standing gastrocnemius-soleus stretching versus night splinting in the treatment of plantar fasciitis. J Foot Ankle Surg. 2002;41(4):221-27. [PMID:12194511] http://dx.doi.org/10.1016/S1067-2516(02)80018-7

14. Batt ME, Tanji JL, Skattum N. Plantar fasciitis: a prospective randomized clinical trial of the tension night splint. Clin J Sport Med. 1996;6(3):158-62. [PMID:8792046] http://dx.doi.org/10.1097/00042752-199607000-00004

15. Mizel MS, Marymont JV, Trepman E. Treatment of plantar fasciitis with a night splint and shoe modification consisting of a steel shank and anterior rocker bottom. Foot Ankle Int. 1996;17(12):732-35. [PMID:8973894]

16. Powell M, Post WR, Keener J, Wearden S. Effective treatment of chronic plantar fasciitis with dorsiflexion night splints: a crossover prospective randomized outcome study. Foot Ankle Int. 1998;19(1):10-18. [PMID:9462907]

17. Probe RA, Baca M, Adams R, Preece C. Night splint treatment for plantar fasciitis. A prospective randomized study. Clin Orthop Relat Res. 1999;(368):190-95. [PMID:10613168]

18. Ryan J. Use of posterior night splints in the treatment of plantar fasciitis. Am Fam Physician. 1995;52(3):891-98, 901-2. [PMID:7653427]

19. Landorf KB, Keenan AM, Herbert RD. Effectiveness of different types of foot orthoses for the treatment of plantar fasciitis. J Am Podiatr Med Assoc. 2004;94(6):542-49. [PMID:15547121]

20. Young CC, Rutherford DS, Niedfeldt MW. Treatment of plantar fasciitis. Am Fam Physician. 2001;63(3):467-74, 477-78. [PMID:11272297]

21. Martin RL, Irrgang JJ, Conti SF. Outcome study of subjects with insertional plantar fasciitis. Foot Ankle Int. 1998; 19(12):803-11. [PMID:9872466]

22. Cohen J. Statistical power analysis for the behavioral sciences. 2nd ed. Hillsdale (NJ): Lawrence Erlbaum Associates; 1988.

23. Budiman-Mak E, Conrad KJ, Roach KE. The Foot Function Index: a measure of foot pain and disability. J Clin Epidemiol. 1991;44(6):561-70. [PMID:2037861] http://dx.doi.org/10.1016/0895-4356(91)90220-4

24. Perry J. Gait analysis: normal and pathological function. Thorofare (NJ): SLACK Inc; 1992.

25. Chang BC, Wang JY, Huang BS, Lin HY, Lee WC. Dynamic impression insole in rheumatoid foot with metatarsal pain. Clin Biomech (Bristol, Avon). 2012;27(2):196-201. 


\section{[PMID:21889242]}

http://dx.doi.org/10.1016/j.clinbiomech.2011.08.005

26. Vela SA, Lavery LA, Armstrong DG, Anaim AA. The effect of increased weight on peak pressures: implications for obesity and diabetic foot pathology. J Foot Ankle Surg. 1998;37(5):416-20, discussion 448-49. [PMID:9798174] http://dx.doi.org/10.1016/S1067-2516(98)80051-3

27. Petrone N, Faggiano E, Meneghello R. Mechanical properties of orthopaedic insoles under cyclic loads and correlation with daily use. Eng Sport. 2006;10:427-32. http://dx.doi.org/10.1007/978-0-387-45951-6_76

28. Portney LG, Watkins MP. Foundations of clinical research: applications to practice. Upper Saddle River (NJ): Pearson/ Prentice Hall; 2009.

29. Tatli YZ, Kapasi S. The real risks of steroid injection for plantar fasciitis, with a review of conservative therapies. Curr Rev Musculoskelet Med. 2009;2(1):3-9. [PMID:19468912] http://dx.doi.org/10.1007/s12178-008-9036-1

30. Tsai WC, Wang CL, Tang FT, Hsu TC, Hsu KH, Wong MK. Treatment of proximal plantar fasciitis with ultrasound-guided steroid injection. Arch Phys Med Rehabil. 2000;81(10):1416-21. [PMID:11030509] http://dx.doi.org/10.1053/apmr.2000.9175

31. Gudeman SD, Eisele SA, Heidt RS Jr, Colosimo AJ, Stroupe AL. Treatment of plantar fasciitis by iontophoresis of $0.4 \%$ dexamethasone. A randomized, double-blind, placebo- controlled study. Am J Sports Med. 1997;25(3):312-16. [PMID:9167809]

http://dx.doi.org/10.1177/036354659702500307

32. DeMaio M, Paine R, Mangine RE, Drez D Jr. Plantar fasciitis. Orthopedics. 1993;16(10):1153-63. [PMID:8255812]

33. Acevedo JI, Beskin JL. Complications of plantar fascia rupture associated with corticosteroid injection. Foot Ankle Int. 1998;19(2):91-97.[PMID:9498581]

34. Sellman JR. Plantar fascia rupture associated with corticosteroid injection. Foot Ankle Int. 1994;15(7):376-81.

[PMID:7951973]

Submitted for publication September 30, 2011. Accepted in revised form April 19, 2012.

This article and all supplementary materials should be cited as follows:

Lee WC, Wong WY, Kung E, Leung AK. Effectiveness of adjustable dorsiflexion night splint in combination with accommodative foot orthosis on plantar fasciitis. J Rehabil Res Dev. 2012;49(10):1557-64. http://dx.doi.org/10.1682/JRRD.2011.09.0181

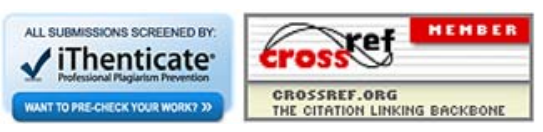

